\title{
AUS WISSENSCHAFT UND PRAXIS
}

\author{
Hellmuth Hecker \\ Staatsangehörigkeit in geteilten Staaten
}

Bei diesem Problem liegt es nahe, vor allem an die deutsche Teilung und die damit verbundenen Staatsangehörigkeitsfragen zu denken. So hautnah und schmerzlich uns diese Frage auch ist, so darf doch nicht übersehen werden, daß sie nicht die einzige ihrer Art ist. Es gibt auch andere geteilte Staaten, die ebenfalls das Problem rivalisierender Staatsangehörigkeitsauffassungen und -regelungen kennen. Gegenüber der immer mehr zunehmenden Fülle von Literatur zur gesamtdeutschen StA fällt auf, daß jene anderen Fälle der StA geteilter Staaten, überhaupt nicht behandelt werden. Soweit ich sehe, finden sich weder konkrete Untersuchungen zu einzelnen dieser Fälle, noch gar Gesamtdarstellungen zur allgemeinen Frage, geschweige denn Vergleiche zur deutschen Literatur.

Der folgende Beitrag versucht, einen ersten kurzen Einstieg in die jeweilige Problematik zu geben und dabei sozusagen das juristische Neuland abzustecken. Dabei sind 4 Fälle zu betrachten, die noch heute aktuell sind. ${ }^{1}$ Der abgeschlossene Fall Vietnam mag hier unberücksichtigt bleiben, auch weil es sich dabei nicht um kalten sondern heißen Krieg gehandelt hatte.

\section{China ${ }^{2}$}

Sowohl die Volksrepublik China (Festland) als auch die Republik China (Taiwan $=$ Formosa) beanspruchen alle Chinesen als ihre Staatsangehörigen, weil sie sich jeweils als die einzig legitime Regierung betrachten. Dieser konkurrierende Alleinvertretungsanspruch perpetuiert die Situation des kalten Krieges.

Die Lage im Staatsangehörigkeitsrecht ist die gleiche wie sie zunächst nach 1949 auch zwischen beiden deutschen Staaten bestanden hatte. Dabei ging die Ähnlichkeit mit der deutschen Lage und der allgemeinen Geltung des RuStAG so weit, daß in beiden Teilen

1 Das zunächst geteilte Mandatsgebiet Palästina und die geteilte Insel Irland sind hier nicht relevant, da sich dort nie 2 rivalisierende Staaten gegenüberstanden.

2 S. LG Düsseldorf v. 29. 9. 1970 (IPRsp. 1970, S. 390) u. E. Tomson, Das StAR der ostasiatischen Staaten, Ffm. 1971, S. 89 (SGS Bd. 32). 
Chinas zunächst das gleiche StAG v. 1929 galt: in Taiwan ausdrücklich, in der Volksrepublik gewohnheitsrechtlich. Die VR hatte nämlich alles vorrevolutionäre Recht generell aufgehoben, wandte es aber an, wenn es dem Marxismus nicht widersprach. So wurde auch das StAG v. 192930 Jahre weiter praktiziert, bis die VR erst 1980 ein eigenes StAG erließ, das aber am Anspruch auf alle Chinesen nichts änderte. So wie in der BRD das alte StAG des Reiches von 1913 weitergalt, so gilt auf Taiwan das alte StAG von 1929; und so wie die DDR ein eigenes StAG erließ, so auch die VR China. Der einzige Unterschied zur deutschen Situation liegt darin, daß nicht nur der legitimistische Staat (Taiwan) einen Gesamtanspruch auf die StA erhebt (wie die BRD), sondern auch die VR China, während die DDR einen solchen Anspruch schon lange aufgegeben hat. Insofern ist die chinesische Situation noch heute radikaler als die deutsche.

\section{Korea $^{3}$}

Nordkorea beanspruchte nach Art. 1 Ziff. 1 seines StAG v. 9. Oktober 1963 alle Personen, die vor Gründung der Volksrepublik Korea (15. 8. 1948) die koreanische StA besaßen. Das bedeutet: Als Korea 1945 von Japan unabhängig geworden war und die Sowjets am 6. September 1945 im Norden eine Volksrepublik ausriefen, gab es auch wieder eine eigene StA. Südkorea hat denselben Anspruch nur versteckter: Nach Art. 4 (jetzt 3) der Verfassungen von 1948, 1962 und 1972 besteht die Republik Korea aus der gesamten Halbinsel Korea. Daher beansprucht es nach Art. 2 seines StAG von 1948 auch die in Nordkorea Geborenen als eigene StA'e.

\section{Kaschmir ${ }^{4}$}

Hier ist die Rechtslage erheblich komplizierter, denn Kaschmir zerfällt in nicht weniger als vier Teile, einen indischen, einen chinesischen, einen pakistanischen und in einen Schutzstaat Pakistans.

a) Der indische Fürstenstaat Jammu und Kaschmir wurde aufgrund eines von der UNO vermittelten Waffenstillstandes am 1. 1.1949 zwischen Indien und Pakistan geteilt, wobei Indien den Löwenanteil erhielt. Indien bürgerte 1954 rückwirkend alle kaschmirischen Untertanen ein sowie alle aus Pakistan zurückkehrenden ständigen Einwohner Kaschmirs. Pakistan erhob keinen Anspruch auf diese Personen, so daß alle früheren ortsansässigen Untertanen Kaschmirs jetzt allein die indische StA besaßen.

3 S. Tomson a.a.O. S. 260 f. und K.-H. Bauer, Die dt. StA, München 1974, S. 202.

4 S. SGS 26, S. 42. Neuere Regelung durch citizenship Amendment Ordinance v. 2. 2. u. Amendment Act. v. 22. 6. 73, abgedruckt in All-Pakistan Legal Decisious 1973, Central S. 200 bzw. 484. Im indischen wie im pakistanischen Kaschmir gilt für das Indigenat aber nach wie vor die gleiche Vorschrift, nämlich eine Notifikation des Maharaja v. 20.4. 1927 betr. Abgrenzung der "State Subjects". 
b) 1959 und 1962 besetzte China $36000 \mathrm{~km}$ des von Indien verwalteten Kaschmir: Es betrachtet die Bewohner allein als chines. StA'e, während Indien sie allein als Inder ansieht. Hier ist also die Lage wie bei den beiden China und Korea.

c) Pakistan erließ 1951 sein erstes StA-Gesetz, welches auch den Bewohnern der von ihm besetzten und annektierten ehem. Fürstenstaaten Kaschmirs in Gilgit und Baltistan, gleicherweise wie allen Bewohnern Pakistans, die StA verlieh. Da Indien nach Art. 4 der Verfassung Kaschmir vom 17. November 1956 das gesamte frühere Kaschmir beansprucht, betrachtet es seitdem diese Personen als Doppelstaatler, offenbar konkurrierend wie bei Korea und China.

"State Subjects" Kaschmirs, die mit pakistan. $\mathrm{Pa}$ im Ausland leben, gelten außerdem seit 1973 als StA'e Pakistans. Das gleiche gilt, wenn Kaschmiris ständig in Pakistan wohnen. Dies können Personen sein, welche außerdem die indische StA als frühere Untertanen Kaschmirs besitzen oder solche, die die Untertanenschaft des Schutzstaates Azad Kaschmir besitzen. Sie sind also ebenfalls Doppelstaater.

d) Der Hauptteil des von Pakistan besetzten Gebietes Kaschmirs wurde nicht annektiert, sondern bildet noch heute einen Schutzstaat Azad Kaschmir: modern ausgedrückt einen assoziierten Staat mit eigener Staatsangehörigkeit. Indien beansprucht auch diese Personen als eigene Staatsangehörige. Da Pakistan die Außenpolitik dieses Staates führt, übernimmt es auch den dipl. Schutz dieser Schutzgenossen im Ausland.

\section{Zypern}

Die Republik Zypern erkennt die Existenz einer "Türkischen Republik Nord-Zypern" mit eigener StA nicht an, die allein von der Türkei anerkannt wird. Zypern beansprucht daher nach wie vor alle Einwohner Nord-Zyperns als seine Staatsangehörigen gemäß dem StAG Nr. 43 vom 28. Juli 1967.

Nord-Zypern erklärte sich am 13. Februar 1975 für unabhängig und gab sich dabei eine Verfassung des "Türkischen Föderativen Staates Zypern", innerhalb einer fingierten Bundesrepublik Zypern. Die in Art. 53 erwähnte StA, die durch Gesetz geregelt werden sollte, wobei ein Ausbürgerungsverbot aufgenommen wurde, ist daher nur als Gliedstaatsangehörigkeit innerhalb einer gesamtzyprischen StA zu betrachten. Die Existenz der gesamtzyprischen StA nach dem StAG von 1967 wurde damit also zunächst noch nicht geleugnet. Eine Doppel-StA bestand hier nicht völkerrechtlich sondern nur staatsrechtlich wie etwa im Verhältnis der slowakischen Glied-StA zur gesamttschechoslowakischen StA.

Dies änderte sich jedoch, als am 15. November 1983 die Türkische Republik Nord-Zypern proklamiert wurde, die jede Bundesstaatlichkeit verneinte. Die in Art. 67 der neuen

5 S. Bergmann/Ferid, Int. Ehe- u. Kinderschaftsrecht, Zypern, 63. Lief. 1979, S. 5 ff.; A.D. Weinberg, Turkish Republic of Northern Cyprus, in: Constitutions of Dependencies and Special Sovereignties 2/1985 und 2/ 1986. 
Verfassung vom 12. März 1985 ausführlich geregelte StA ist daher eine KonkurrenzStA, die eine gesamtzyprische StA leugnet, so wie die DDR eine gesamtdeutsche StA leugnet. Die StA erwerben vor allem diejenigen Zyprioten, die am 15. November 1983 im Nordstaat wohnten oder vorher die StA des bisherigen Türkischen Gliedstaates erworben hatten (wohl durch Einbürgerung?). Auch für künftig geborene Personen wird die StA in Art. 67 geregelt. 